\title{
Up and Away in the Potential Landscape of Diatomic Molecule Potential Energy Curves
}

\section{William C. Stwalley, Departments of Physics and Chemistry, University of Connecticut, Storrs, CT 06269-3046}

\begin{abstract}
The understanding of the potential curves of a given molecule has expanded in many ways during my last 52 years as an experimental and theoretical molecular chemist/physicist in graduate school in Chemistry at Harvard and in both the Chemistry and Physics Departments at University of Iowa and University of Connecticut. This expansion has been $u p$ in energy and vibrational and rotational quantum numbers and away from $\mathrm{R}_{\mathrm{e}}$ to long range as well. It is clear that Prof. Robert Le Roy, who I have known since the late 1960s, has made many important and greatly appreciated contributions to the landscape I describe below from my personal perspective, especially with regard to long range molecules and solution of the radial Schrödinger equations and related calculations.
\end{abstract}




\section{Introduction}

The potential energy, $\mathrm{V}(\mathrm{R})$, as a function of internuclear distance, $\mathrm{R}$, for a given electronic state of a diatomic molecule in the Born-Oppenheimer Approximation [fast light electrons, slow heavy nuclei] is an old but extremely valuable concept. Experimentally, diatomic spectroscopy (especially rovibronic, but also rovibrational and rotational) has provided the most accurate information, but also atom-atom scattering and collisional properties of atomic gases including bulk and transport can be used to determine the potential energy curve. Theoretically, $a b$ initio and a wide variety of other theoretical techniques have been applied for calculation of full potential curves, long range potentials, Rydberg state potentials, exchange interaction potentials involving sets of states, etc. Here I will emphasize diatomic spectroscopy in comparison with appropriate theory but with occasional related comments, and will stay below the molecular ion asymptote in energy.

With regard to diatomic spectroscopy, the detailed approaches evolved in various areas of potential energy curves during the past century. As described in more detail below, the earliest observation of vibrational structure in potential energy curves of which I am aware was published for $\mathrm{Na}_{2}$ and $\mathrm{K}_{2}$ in 1874 [1], but not clearly explained until nearly a half century later (1924-27). The near constant vibrational spacings in the $A$ and $B$ states of $\mathrm{Na}_{2}$ and $\mathrm{K}_{2}$ must have suggested first electronic excitation as in atoms, but also vibrational motion as in a slightly anharmonic oscillator. Similar later observations for the isotopic variants (isotopologues) of molecules such as $\mathrm{H}_{2}$ and $\mathrm{Li}_{2}$ must have suggested the reduced mass dependence of these diatomic molecular oscillators as well, especially given the concept of a mass-reduced vibrational quantum number, $\eta$ [2], where $\eta=(v+$ $1 / 2)(\mu)^{-1 / 2}$. Likewise, the reduced mass dependence of the behavior of such diatomic molecular rotors can written in terms of a mass-reduced rotational quantum number, $\xi[2]$, where $\xi=\mathrm{J}(\mathrm{J}+1)(\mu)^{-1}$.

The key equation for the nuclear motion in a diatomic molecule is the radial Schrödinger equation (in atomic units: $\mathrm{h} / 2 \pi=|\mathrm{e}|=\mathrm{m}_{\mathrm{e}}=1$ ):

$$
\mathrm{H}_{\mathrm{R}} \psi_{\mathrm{vJ}}(\mathrm{R})=\mathrm{E}_{\mathrm{vJ}} \psi_{\mathrm{vJ}}(\mathrm{R})
$$


where $\mathrm{H}_{\mathrm{R}}=\left[-(1 / 2 \mu) \partial^{2} / \partial \mathrm{R}^{2}+\xi / 2 \mathrm{R}^{2}+\mathrm{V}(\mathrm{R})\right]$ and the angular Schrödinger equation solutions are the spherical harmonics $\mathrm{Y}_{\mathrm{JM}}(\Theta, \Phi)$, as in other central force problems like the $\mathrm{H}$ atom. Note that in many contexts, we can consider $\mathrm{V}+\mathrm{J}(\mathrm{J}+1) / 2 \mu \mathrm{R}^{2}$ to be an effective potential, $\mathrm{U}_{\mathrm{J}}$, a function of $\mathrm{J}$ or better yet $\xi=\mathrm{J}(\mathrm{J}+1) / \mu$. Moreover, $\mathrm{U}_{\mathrm{J}}$ or $\mathrm{U}_{\xi}$ can incorporate not only the $\mu$ dependence in rotation, but also the $\mu$ dependence for breakdown of the Born-Oppenheimer approximation.

Since joining Prof. Dudley Herschbach's research group in 1964, I have had an enchanting and continuing tour [52 years so far] by going up in energy E and away in internuclear distance $\mathrm{R}$ through the potential energy landscape of many diatomic molecules (including multiple isotopologues to study the effects of the variation in $\mu$ ), specializing in the simple hydrogen-like diatomics, e.g. $\mathrm{H}_{2}, \mathrm{LiH}, \mathrm{Li}_{2}, \mathrm{Na}_{2}, \mathrm{~K}_{2}$, $\mathrm{KRb}, \mathrm{Rb}_{2}$, and $\mathrm{Cs}_{2}$, but also visiting some other simple systems such as $\mathrm{HgH}$ and $\mathrm{Mg}_{2}$. I have some interest in the potentials of diatomic positive and negative ions as well, but will not include these here, except for a brief mention in Section V. In addition, there are many cases where a single electronic state incorporates electronic configurations of multiple different characters, giving rise to multiple extrema in the potential, but a general prescription is not available. I will incorporate some limited comments on spin-orbit coupling, but will generally omit the complexities of hyperfine coupling.

In addition to going $\boldsymbol{u p}$ and $\boldsymbol{a w a y}$ in $\mathrm{E}$ and $\mathrm{R}$, I have also been exploring the region of vibrational quantum numbers $\mathrm{v}$ and $\eta$, and of rotational quantum numbers $J$ and $\xi$, all of which have finite limits except for the "Heavy Rydberg" (ion pair) electronic states with a long range $1 / \mathrm{R}$ potential. The range of reduced masses $\mu$ is also limited, with the isotopic hydrogen and hydride diatomics showing the greatest variation in the value of $\mu$.

I have also found ultracold $(\mathrm{T}<1 \mathrm{mK})$ molecular spectroscopy $[3,4]$ to be a fascinating alternative to ordinary diatomic spectroscopy, where one often begins with a very broad thermal rovibrational distribution in the ground electronic state. Using a supersonic molecular beam, one can collapse the thermal population to temperatures of $\sim 1-10 \mathrm{~K}$, often with most population in the lowest $\mathrm{v}=0$ level and 
typically with $\mathrm{J}<10$. Using ultracold photoassociation [4-6] and other techniques, one can form ultracold molecules in a variety of vibrational levels, often with the maximum $\mathrm{v}$ observed very near dissociation, and with very low rotational quantum numbers (typically $\mathrm{J}<6$ ). Recently my collaborators and I [7] have also studied the power of combining ultracold molecule spectra and molecular beam spectra to unravel complex spectra and to seek efficient Raman pathways from, for example, a primarily triplet $\mathrm{KRb}$ "Feshbach molecule" near dissociation and the lowest rovibrational level of the $X$ ground state, $X(0,0)$, via a mixed singlet-triplet excited state.

In the specific Sections below I will briefly cover the following regions of potentials, with the designation of each on the graphical abstract indicated:

I. Short Range Chemically Bound Levels[S]

II. Long Range Weakly Bound Levels[L]

III. Long Range Purely Repulsive Continuum Levels[C]

IV. Rydberg Levels Based on Short Range Chemically Bound Ions[RS]

V. Rydberg Levels Based on Long Range Weakly Bound Ions[RL]

VI. Long Range "Heavy" Rydberg Levels Based on Atomic Ion Pairs[HR]

VII. Long Range Rydberg Levels Based on an Atom @ Rydberg Atom[@R]

\section{Short Range Chemically Bound Levels[S]}

The primary spectroscopic tool for determining the potential near its minimum at $R_{e}$, where the reference energy is normally taken to be $E=0$, is the so-called Dunham expansion [8]:

$$
\mathrm{V}_{\mathrm{D}}(\mathrm{R})=\mathrm{a}_{00}\left(\left(\mathrm{R}-\mathrm{R}_{\mathrm{e}}\right) / \mathrm{R}_{\mathrm{e}}\right)^{2}\left(1+\sum_{\mathrm{i}=1-\infty} \mathrm{a}_{\mathrm{i} 0}\left(\left(\mathrm{R}-\mathrm{R}_{\mathrm{e}}\right) / \mathrm{R}_{\mathrm{e}}\right)^{\mathrm{i}}\right)
$$

Later variants used a variable such as $\left(\mathrm{R}-\mathrm{R}_{\mathrm{e}}\right) / \mathrm{R}[9]$ and $\lambda(\mathrm{p})=\operatorname{sgn}(\mathrm{p})\left(1-\left(\mathrm{R}_{\mathrm{e}} / \mathrm{R}\right)^{\mathrm{p}}\right)$, where $\operatorname{sgn}(p)=+1$ for $p>0$ and -1 for $p<0[10]$ to replace $\left(R-R_{e}\right) / R_{e}$. Dunham semiclassically derived the relations 


$$
\mathrm{E}(\mathrm{v})=\sum_{\mathrm{i}=0-\infty} \mathrm{Y}_{\mathrm{i} 0}(\mathrm{v}+1 / 2)^{\mathrm{i}} \text { for pure vibration }
$$

and

$$
\mathrm{E}(\mathrm{v}, \mathrm{J})=\sum_{\mathrm{i}=0-\infty, \mathrm{j}=0-\infty} \mathrm{Y}_{\mathrm{ij}}(\mathrm{v}+1 / 2)^{\mathrm{i}}(\mathrm{J}(\mathrm{J}+1))^{\mathrm{j}} \text { for vibration and rotation }
$$

with the $Y_{i 0}$ constants determining the potential (for $\mathrm{J}=0$ ) and the $\mathrm{Y}_{\mathrm{ij}}$ constants for all $\mathrm{J}$ including 0 determining the effective potential

$$
\mathrm{U}_{\mathrm{J}}(\mathrm{R})=\mathrm{V}(\mathrm{R})+\mathrm{J}(\mathrm{J}+1) / 2 \mu \mathrm{R}^{2}
$$

Kilpatrick [11] showed that the above Dunham equations could also be derived quantum mechanically. It is also worth noting that equation (4) can be written [2] in terms of the mass-reduced quantum numbers $\eta$ and $\xi$ and mass-reduced Dunham coefficients $Z_{\mathrm{ijk}}$ :

$$
E(\eta, \xi, \mu)=\sum_{\mathrm{i}=0-\infty, \mathrm{j}=0-\infty, \mathrm{k}=0-\infty} \mathrm{Z}_{\mathrm{ijk}} \eta^{\mathrm{i} \xi \mathrm{j}}(1 / \mu)^{\mathrm{k}}
$$

The original cases considered in [2] included the isotopologues of $\mathrm{LiH}, \mathrm{H}_{2}$, and HF. However, the unusual molecule, $\mathrm{HgH}$, which is discussed below, is a particularly interesting and challenging case, where the simplest theory would incorrectly predict a "long-range molecule" such as HHe, HAr, and HXe.

An important offshoot of the Dunham and related methods is the RydbergKlein-Rees (RKR) method [12-15] by which the vibrational and rotational data up to a particular vibrational level (call it $\mathrm{v}_{\max }$ ) can be combined in two semiclassical equations to calculate the two quantities $\left(\mathrm{R}_{\mathrm{v}, \mathrm{J}-}-\mathrm{R}_{\mathrm{v}, \mathrm{J}+}\right)$ and $\left(\left(\mathrm{R}_{\mathrm{v}, \mathrm{J}-}\right)^{-1}-\left(\mathrm{R}_{\mathrm{v}, \mathrm{J}+}\right)^{-1}\right)$, which then yield the two classical turning points $\mathrm{R}_{\mathrm{v}, \mathrm{J}-}$ (inner) and $\mathrm{R}_{\mathrm{v}, \mathrm{J}+}$ (outer) for levels from $\mathrm{v}=0$ up to some $\mathrm{v}_{\max }$. In addition, for $\mathrm{J}=0$, the quantity $\mathrm{B}_{\mathrm{e}}$ can be used to determine $R_{e}$ at the minimum of the potential. For $J>0$, a value for $R_{e J}$ can be similarly determined. For $\mathbf{J}=0$, this then completes the ordinary potential energy curve defined by $2 \mathrm{v}_{\max }+1$ points, while for finite $\mathrm{J}$ it is the effective potential energy curve with $2 \mathrm{v}_{\max }+1$ points. Using mass-reduced scaling, the turning points can easily be interpolated by simply using a non-physical mass; e.g. a non-physical mass of $100 \mu$ would give $10 \mathrm{x}$ as many turning points. 
In order to obtain even better agreement between the potential and the quantum mechanically calculated spectroscopic properties, an "Inverted Perturbation Approach" (IPA) [16-18] has been developed which uses the differences between the initial spectroscopic data and the same data calculated quantum mechanically from the preliminary potential (e.g. RKR). This approach can converge to a significantly improved "quantum" potential, especially for diatomics of light reduced mass.

A conceptually similar "Direct Potential Fit"(DPF) approach was pioneered and extended by Le Roy et al. [19-22], using various potential models such as "expanded Morse oscillator", "Morse/Lennard-Jones", and "Morse/Long-Range" [21]. However, while this approach works very well for many states of molecules of high interest to me such as $\mathrm{Rb}_{2}$ [20] and $\mathrm{Li}_{2}[22]$, I believe a more elaborate model would be required for the curious example of the $\mathrm{HgH}$ ground state discussed below, where two different electronic configurations are mixed in different adjacent regions of the ground state potential.

It is also worth briefly noting the evolution of increasingly accurate numerical solutions of the radial Schrödinger equation, especially at long range, pioneered by Le Roy et al., from his early review [23] to the current suite of LEVEL and related programs (BCONT, RKR1-16, dParFit16, DPo0tFit16, betaFit16, and APHSHFT) [24].

With regard to the rather fascinating molecule $\mathrm{HgH}$, Bernstein [25] made the assumption that the outer portion of the ground state diatomic potential of $\mathrm{HgH}$ could be represented as $-\mathrm{C}_{6} \mathrm{R}^{-6}$, and then predicted the previously observed rotational predissociation of the $\mathrm{HgH}$ and $\mathrm{HgD}$ molecules by calculating the locus of barrier maxima for the effective potentials of $\mathrm{HgH}$ and $\mathrm{HgD}$. In other words, Bernstein had assumed $\mathrm{HgH}$ was a "long-range molecule" where no chemical bonding was involved, despite the fact that the $\mathrm{R}_{\mathrm{e}}$ value for $\mathrm{HgH}$ was only $1.74 \AA$. This assumption had led to a reasonable fit to the rotational predissociation data for $\mathrm{HgH}$ and $\mathrm{HgD}$, as well as a reasonable value for the $\mathrm{C}_{6}$ coefficient at long range. 
In fact, Mulliken [26] had previously argued that there was a partial chemical bonding in the $\mathrm{X}$ ground electronic state of $\mathrm{HgH}$ because of the mixing of the longrange non-bonding $\mathrm{X}^{2} \Sigma^{+}$state correlating to the $\mathrm{Hg}\left(6 \mathrm{~s}^{2}{ }^{1} \mathrm{~S}\right)+\mathrm{H}\left(1 \mathrm{~s}{ }^{2} \mathrm{~S}\right)$ asymptote with the excited $2^{2} \Sigma^{+}$state correlating to the $\mathrm{Hg}\left(6 \mathrm{~s} 6 \mathrm{p}{ }^{3} \mathrm{P}\right)+\mathrm{H}\left(1 \mathrm{~s}{ }^{2} \mathrm{~S}\right)$ asymptote, an argument repeated by Herzberg [27], with a related Figure 165 for the related case of $\mathrm{BeH}$. This mixing has been confirmed by later theoretical calculations, e.g. by Hay et al. [28]; see also the recent paper by Sasmal et al. [29] where many other $\mathrm{HgH}$ calculations are referenced. Data from molecular beam scattering experiments also support the idea of a shorter distance and a stronger bond than in a true "long-range molecule" species (see e.g. Fig. 2 in [30]).

Finally, a distance criterion for "long-range" where the long-range $\mathrm{C}_{6 \mathrm{AB}}$ term in the potential difference from the asymptote is accurate to $\sim 10 \%$ has been proposed by $[23,31]$ :

$$
\mathrm{R}_{\mathrm{LR}}=2\left[\left\langle\mathrm{r}^{2}\right\rangle_{\mathrm{A}}{ }^{1 / 2}+\left\langle\mathrm{r}^{2}\right\rangle_{\mathrm{B}}{ }^{1 / 2}\right] .
$$

This Long Range or Le Roy criterion gives $\mathrm{R}_{\mathrm{LR}}=4.84 \AA$ for ground state $\mathrm{HgH}$ (see Appendix $C$ of [2]), which is far greater than $R_{e}=1.74 \AA$, and also greater than the range (2.3-3.4 $\AA$ in $[2,25]$ ) of the barrier maxima in the effective potential curves for the rotational predissociation of the $v=0-3$ levels of $\mathrm{HgH}$. A modified $\mathrm{R}_{\mathrm{LR} \Lambda}$ criterion for electronic states with a nonzero electronic orbital angular momentum projection on the internuclear axis, $\Lambda=\left|\mathrm{m}+\mathrm{m}^{\prime}\right|>0$ was proposed in [32]:

$$
\left.\left.\mathrm{R}_{\mathrm{LR \Lambda}}=2(3)^{1 / 2}\right]|<\mathrm{nlm}| \mathrm{z}^{2}\left|\mathrm{nlm}>_{\mathrm{A}}\right|^{1 / 2}+\mid<\text { n'l'm }^{\prime}\left|\mathrm{z}^{2}\right| \mathrm{n}^{\prime} \mathrm{l}^{\prime} \mathrm{m}^{\prime}>\left._{\mathrm{B}}\right|^{1 / 2}\right],
$$

with reasonable agreement with potential energy curves for excited states of $\mathrm{Na}_{2}$.

Moreover, the potential of the $\mathrm{X}$ state of $\mathrm{HgH}$ has been determined experimentally by an isotopologically combined potential, labelled IC1 in [2]. It might first be noted that there are only five observed levels of the $\mathrm{X}$ state of $\mathrm{HgH}$, $\mathrm{v}=0-4$. A Dunham fit (polynomial in $\left(\mathrm{R}-\mathrm{R}_{\mathrm{e}}\right) / \mathrm{R}_{\mathrm{e}}$ ) produces unsatisfactory results; in particular, the polynomial reaches a maximum at $\mathrm{v}+1 / 2=4.3$, i.e. at $\mathrm{v}=3.8$, below the highest observed vibrational level, $v=4$. Quite unphysically, this suggests that the vibrational energy decreases when $v$ increases! However, given 
the Born-Oppenheimer approximation, data for the vibrational levels of the $\mathrm{HgD}$ and $\mathrm{HgT}$ isotopologues can be used as well (The spread in mass of $\mathrm{Hg}$ isotopes is not significant for these small reduced mass species). Such a fit including the $\mathrm{HgH}, \mathrm{HgD}$ and $\mathrm{HgT}$ data does not show a maximum below the highest energy $\mathrm{HgH}$ vibrational level and can be used to generate this much more satisfactory IC1 potential.

This IC1 potential is particularly interesting in the region $3-5 \AA$, where the slope of the potential initially decreases as is usual, but then increases, and finally decreases again with the slope expected from the long range $\mathrm{C}_{6}$ and $\mathrm{C}_{8}$ terms, as shown in Fig. 11 of [2]. This is interpreted as a "psuedocrossing shoulder" where the mixing of the $\mathrm{X}^{2} \Sigma^{+}$and $2^{2} \Sigma^{+}$states is rapidly decreasing with increasing $\mathrm{R}$. This change in slope gives a very interesting set of barrier maxima in the effective potentials; for $\mathrm{K}=8-13$ there are two distinct barrier maxima, a short range one in the region of the shoulder (higher for $K=10-13$ ), and a long range one (higher for $\mathrm{K}=8-9$ ), as shown in Fig. 12 of [2].

Finally, it is noteworthy that three experimental studies of $\mathrm{H}+\mathrm{Hg}$ and $\mathrm{D}+\mathrm{Hg}$ total elastic scattering cross sections as a function of energy from $2 \times 10^{-4}-7 \times 10^{-2}$ atomic units agree very well with theoretical calculations using the IC1 potential and $\boldsymbol{n o}$ adjustable parameters [33], including 4 and 5 Glory oscillation maxima for $\mathrm{H}$ and for $\mathrm{D}$, respectively. There is also some agreement concerning so-called orbiting resonances, the scattering equivalent of rotationally predissociative levels (e.g. the $\mathrm{HgH} \mathrm{v}=3, \mathrm{~K}=9$ level). Using the improved observation of this orbiting resonance, Köhler et al. [34] significantly improved the dissociation energy and the $\mathrm{HgH} \mathrm{X}$ state potential in the psuedocrossing region, with a better defined shoulder in the potential (Fig. 5). This potential based on a combination of spectra and scattering [34] is still the best potential for ground state $\mathrm{HgH}$ of which I am aware.

\section{Long Range Weakly Bound Levels[L]}

There are two ways in which one can find long range weakly bound levels: in a van der Waals molecule, such as $\mathrm{He}_{2}$ [35] or $\mathrm{Mg}_{2}$ [36-38], where there is no 
significant chemical bonding; or in the highest levels of a chemically bound potential, such as the $\mathrm{X}$ state of $\mathrm{Li}_{2}$ [39] and the $\mathrm{B}$ state of $\mathrm{H}_{2}$ [40, 41], where the vast majority of the vibrational period is spent within the long range region (say $\mathrm{R} \gg>\mathrm{R}_{\mathrm{LR}}$ ). It is interesting that if one simply observes the long range levels without knowing their short range correlations, one cannot easily tell which case one is dealing with. For example, using ultracold photoassociation, we observed many vibrational levels of each of three nearly parallel long range potentials, which all correlated with the $\mathrm{K}\left(4 \mathrm{~s}^{2} \mathrm{~S}\right)+\mathrm{Rb}\left(5 \mathrm{p}{ }^{2} \mathrm{P}_{1 / 2}\right)$ asymptote of $\mathrm{KRb}$ [42]. In fact two of the three potentials correlated with a weakly chemically bound short range state and one correlated with the much more strongly chemically bound short range state, as described below.

The breakthrough in treating the effect of an attractive leading long-range term in the potential, $-\mathrm{C}_{\mathrm{n}} \mathrm{R}^{-\mathrm{n}}$, was the work of LeRoy and Bernstein [43], who published the semiclassical equation

$$
\varepsilon(v)=a_{n}^{\infty}\left(v_{D}-v\right)^{2 n /(n-2)}
$$

where $\varepsilon(\mathrm{v})$ is the binding energy of vibrational level $\mathrm{v}$ measured down from the appropriate dissociation limit (taken to be 0 ); $\mathrm{a}_{\mathrm{n}}{ }^{\infty}$ is a constant dependent only on $\mathrm{n}$, the reduced mass $\mu$, and the $C_{n}$ coefficient; and $v_{D}$ is the vibrational quantum number at dissociation, which is generally not an integer. A similar but less precise derivation of equation (8) was later published [40]. Table 3 in [44] summarizes the many other semiclassical quantities that behave similarly for long range molecules in the limit that $\mathrm{v} \rightarrow \mathrm{v}_{\mathrm{D}}$, including the vibrational spacing, the rotational constant, the centrifugal distortion constant, the classical vibrational period, the density of states, the outer turning point, expectation values of powers of $\mathrm{R}$, the average potential energy and average kinetic energy, etc. (see also [45, 46]). For example, the power $\mathrm{P}$ in $\left(\mathrm{v}_{\mathrm{D}}-\mathrm{v}\right)^{\mathrm{P}}$ depends only on $\mathrm{n}$ and is the same $\mathrm{P}=$ $(n+2) /(n-2)$ [or $P=2$ for $n=6$ ] for the vibrational spacing, the kinetic energy, and the potential energy and is the same $P=-(n+2) /(n-2)$ [or $P=-2$ for $n=6$ ] for the density of states and the classical vibrational period. 
Let us briefly describe the following molecular cases:

For ${ }^{4} \mathrm{He}_{2}$, Uang and I [35] found that within experimental uncertainties, there is probably only one level $(\mathrm{v}=0, \mathrm{~J}=0)$ in the ground $\mathrm{X}$ state and no bound states for the 3-4 and 3-3 isotopologues, and that the ${ }^{4} \mathrm{He}_{3}$ "Efimov" trimer is not bound. More recent studies, summarized in [47], some of which have included retardation effects in the potential, which [35] did not, reached the same conclusions that there was a single $\mathrm{v}=0, \mathrm{~J}=0$ level in ${ }^{4} \mathrm{He}_{2}$ with an even larger outer turning point and there was no ${ }^{4} \mathrm{He}_{3}$ Efimov trimer state.

For $\mathrm{Mg}_{2}$ [36-38], data on $\mathrm{v}=0-12$ in the ground state was used first to predict $\mathrm{D}_{\mathrm{e}}$ and $\mathrm{C}_{8}$ given a theoretical value of $\mathrm{C}_{6}$, and then to assign a $\mathrm{v}=13$ band and predict the additional unobserved $\mathrm{v}, \mathrm{J}$ levels up to $\mathrm{v}=18, \mathrm{~J}=2$.

For $\mathrm{Li}_{2,}$ [40] compared a normal Birge-Sponer extrapolation to obtain $\mathrm{D}_{\mathrm{e}}$ with an extrapolation that included the correct behavior of the long range levels near dissociation; the change increased the $\mathrm{D}_{\mathrm{e}}$ value by $\sim 14 \mathrm{~cm}^{-1}$.

For the $\mathrm{B}^{1} \Sigma_{\mathrm{u}}{ }^{+}$state of $\mathrm{H}_{2}$, I assigned long range levels of the B state [41] from the published spectra [48]. Using equation (8), it was possible to assign spectra of levels $\mathrm{v}=36-39$ and predict all additional levels $\mathrm{v}=40-43$, as well as determine $\mathrm{v}_{\mathrm{D}}$ $=43.26+/-0.04$ for the $\mathrm{B}$ state. This allowed a determination of an improved $\mathrm{D}_{0}=$ $36118.6+/-0.5 \mathrm{~cm}^{-1}$ for the $\mathrm{X}$ ground state of $\mathrm{H}_{2}$. In [42], these data were used to determine the full potential energy curve for the B state of $\mathrm{H}_{2}$.

For $\mathrm{KRb}$, consider three long range states with near parallel long range potentials (in Hund's case (c) notation the $2\left(0^{+}\right), 2\left(0^{-}\right)$and 2(1) states) [43]. In a LeRoyBernstein fit, the three states have almost the same $\mathrm{C}_{6}$ values (in $10^{5}$ atomic units, $1.06+/-0.06,1.01+/-0.04$, and $1.01+/-0.05$, respectively) and $\mathrm{v}_{\mathrm{D}}$ values $(34.1+/$ $0.1,33.6+/-0.2$, and $33.8+/-0.1$, respectively). However, the $2\left(0^{+}\right)$state correlates to the strongly bound $\mathrm{A}^{1} \Sigma^{+}$state with significant ion-pair character, while the $2\left(0^{-}\right)$and $2(1)$ states correlate to the more weakly chemically bound $2{ }^{3} \Sigma^{+}$ state. 
We also note the important relationship of the average and the difference between potentials with a short range minimum and long range potentials dissociating to the same asymptote (e.g. two ground state alkali atoms) due to dispersion and to exchange, respectively. For the alkali dimers in the $\mathrm{X}^{1} \Sigma_{\mathrm{g}}{ }^{+}$and a ${ }^{3} \Sigma_{\mathrm{u}}{ }^{+}$states, we have examined theoretical and experimental results for $\mathrm{Li}_{2}$ [49]; $\mathrm{Na}_{2}$ [50]; $\mathrm{K}_{2}$ [51, 52]; a heteronuclear example, $\mathrm{NaK}$ [53]; and a summary [54].

We also wish to note the development of a novel kind of bound long range molecule, the "pure long range molecule" [55], where the effects of angular momentum (spin-orbit) coupling are combined with long range terms in the potential, e.g. the $\mathrm{C}_{3}$ terms, to give potential wells of $\sim 1.91 \mathrm{~cm}^{-1}$ and $\sim 0.16 \mathrm{~cm}^{-1}$ at very large distances, $\sim 37.9 \AA$ and $\sim 52.5 \AA$, respectively, for two bound states for $\mathrm{Na}_{2}\left(0_{\mathrm{g}}{ }^{-}\right.$and $1_{\mathrm{u}}$ in Hund's case (c) notation) slightly below the $\mathrm{Na}\left(3 \mathrm{~s}{ }^{2} \mathrm{~S}\right)+\mathrm{Na}(3 \mathrm{p}$ ${ }^{2} \mathrm{P}_{3 / 2}$ ) asymptote. A small maximum is also produced in another $1_{\mathrm{u}}$ state, slightly above the $\mathrm{Na}\left(3 \mathrm{~s}^{2} \mathrm{~S}\right)+\mathrm{Na}\left(3 \mathrm{p}^{2} \mathrm{P}_{1 / 2}\right)$ asymptote. These extrema were first predicted in atomic line broadening following the observation of extrema on the wings of the $\mathrm{Rb}$ and $\mathrm{Cs}$ resonance lines [56]. In a special issue in honor of Friedrich Hund decades later [57], observations using ultracold photoassociative spectroscopy of these pure long range molecules were first reviewed for ${ }^{23} \mathrm{Na}_{2}$ in the $0_{\mathrm{g}}{ }^{-}$state [58], for ${ }^{39} \mathrm{~K}_{2}$ in the $0_{\mathrm{g}}{ }^{-}$state [59]( and later in the $1_{\mathrm{u}}$ state [60]), and in ${ }^{85} \mathrm{Rb}_{2}$ in the $0_{\mathrm{g}}{ }^{-}$ state [61]. For detailed information and plots of the short range, intermediate, and long range potentials for both $\mathrm{Rb}_{2}$ and $\mathrm{KRb}$, see [62]. To the best of my knowledge, the small maximum in the lower $1_{\mathrm{u}}$ potential has not been directly observed experimentally. It might also be noted that pure long range minima were not found at the $\mathrm{K}\left(4 \mathrm{~s}^{2} \mathrm{~S}\right)+\mathrm{K}\left(5 \mathrm{p}{ }^{2} \mathrm{P}_{1 / 2}\right)$ and $\mathrm{K}\left(4 \mathrm{~s}^{2} \mathrm{~S}\right)+\mathrm{K}\left(5 \mathrm{p}^{2} \mathrm{P}_{3 / 2}\right)$ asymptotes, although many other potentials did have a pure long range maximum (see [5], e.g. Figure 31).

Finally let me note the possibility of studying an entire effective potential curve at finite $\mathrm{J}>0$ using a single laser-induced fluorescence series [63]. An interesting 
example of this is [64], where a $\mathrm{Kr}+$ laser line excited the $\mathrm{v}^{\prime}=43, \mathrm{~J}^{\prime}=50$ level of the $\mathrm{A}^{1} \Sigma_{\mathrm{u}}{ }^{+}$state of $\mathrm{Na}_{2}$. In this case, fluorescence occurs to $\mathrm{J} "=49$ and 51 levels of $\mathrm{v}$ " $=3-56$ vibrational levels of the $\mathrm{X}^{1} \Sigma_{\mathrm{g}}{ }^{+}$state, basically all of the levels in the $\mathrm{U}_{49}$ and $\mathrm{U}_{51}$ effective potentials (except $\mathrm{v} "=0,1$, and 2 ). There is significant broadening in the $\mathrm{v} "=55$ and 56 levels from tunneling through effective potential barriers and also three continuum oscillations observed, which are well predicted from the experimentally based potentials.

\section{Long Range Purely Repulsive Continuum Levels[C]}

In examining the long range potentials of an alkali metal diatomic such as $\mathrm{Rb}_{2}$ or $\mathrm{KRb}$, e.g. at the first two homonuclear excited asymptotes, $5 \mathrm{~s}+5 \mathrm{p}_{1 / 2}$ and $5 \mathrm{~s}+$ $5 p_{3 / 2}$, or at the first four heteronuclear excited asymptotes, $4 s+5 p_{1 / 2}, 4 s+5 p_{3 / 2}$, $4 p_{1 / 2}+5 s$, and $4 p_{3 / 2}+5 s$, one finds that about half the potentials are repulsive and about half are attractive at long range in Figures 3 and 4 of [63]. However, as noted above for pure long range molecules, sometimes the slope of the potential changes from repulsive to attractive or vice versa. Looking at the same potentials at intermediate range in Figures 5 and 6 and Tables 1 and 2 of [63], one sees additional complexities, with many potentials having 2, 3 or even 4 extrema!

A very different spectrum appears in [65], where a two-photon absorption to an autoionizing state in $\mathrm{Li}_{2}$ is resonantly enhanced by the intermediate $1{ }^{3} \Pi_{\mathrm{g}}$ state. We have not studied the $\mathrm{Li}_{2}$ state since, but we have studied all $4 \Omega$ components $(\Omega$ $=0^{+}, 0^{-}, 1$ and 2) of the analogous $1^{3} \Pi_{\mathrm{g}}$ state in $\mathrm{Rb}_{2}[66]$ and the analogous $2^{3} \Pi$ state in $\mathrm{KRb}$ [67]. Not only does each $\Omega$ component potential for both $\mathrm{Rb}_{2}$ and $\mathrm{KRb}$ have a significant well at $\sim 5 \AA$, there is a much longer range well in the $\Omega=$ $0^{-}$component potential only at $\sim 9 \AA$. Note that all these levels in $\mathrm{Li}_{2}, \mathrm{Rb}_{2}$, and $\mathrm{KRb}$ are not bound levels, but rather quasibound levels in the continuum.

Another challenge is to determine the potential of a lower state by bound $\rightarrow$ free emission from a higher state. This has been used to determine the repulsive 
wall of the a ${ }^{3} \Sigma_{\mathrm{u}}{ }^{+}$state of NaK [68]. It includes a discussion of the classical Franck-Condon approximation in Figure 4, where a so-called Mulliken Difference Potential is used to estimate the relative weight of different internuclear distances in a transition involving a continuum. Further arguments and examples of Mulliken Difference Potentials are discussed in [62].

\section{Rydberg Levels Based on Short Range Chemically Bound Molecular Ions[RS]}

Long before spectroscopists moved away to long range, as described above, they moved $\boldsymbol{u p}$ to higher energies. The simplest way to do this was in analogy with the Rydberg states of atoms, considering a (molecular ion) ${ }^{+1}$ with an outer (electron) ${ }^{-1}$, e.g. the $\mathrm{H}_{2}$ excited molecule formed from the $\mathrm{X}$ ground state of $\mathrm{H}_{2}{ }^{+}$ and a Rydberg electron e'. [27], p. 322f, includes a nice discussion of a "single electron in an axially symmetric electric field". The update of the famous Table 39 of [27], namely [69], contains 169 references for $\mathrm{H}_{2}$, very many involving these Rydberg states.

The potential energy curves of many such Rydberg states formed from a ground state hydrogenic atom (e.g. $\mathrm{H}, \mathrm{Li}, \mathrm{Na}, \mathrm{K}, \mathrm{Rb}, \mathrm{Cs}$ ) and an excited hydrogenic state of the same atom $\mid n l \Lambda>$ can be written in atomic units of energy [70] as

$$
\mathrm{V}_{\mathrm{nl} \Lambda}(\mathrm{R})=\mathrm{V}^{+}(\mathrm{R})-1 / 2\left[\mathrm{n}-\delta_{\mathrm{nl} \Lambda}(\mathrm{R})\right]^{-2}
$$

where $\mathrm{n}$ is the principal quantum number, $\delta_{\mathrm{nl} \Lambda}(\mathrm{R})$ is the quantum defect as a function of $R$, and $V^{+}(R)$ is the potential energy curve of the ground electronic state of the corresponding molecular ion.

My efforts have been relatively modest in this area. I have long been interested in the Rydberg states of diatomic alkali metals, e.g. of $\mathrm{K}_{2}$ (see [71] and references therein), and of $\mathrm{Na}_{2}$ (see [72, 73]). The latter reported triple resonance spectroscopy to reach $n$ levels up to 115 and to determine the ionization energy of $\mathrm{Na}_{2}$ to $+/-0.013 \mathrm{~cm}^{-1}$ and the dissociation energy of $\mathrm{Na}_{2}{ }^{+}$to $+/-0.014 \mathrm{~cm}^{-1}$. However, the next three sections discuss other kinds of Rydberg states of significant interest. 


\section{Rydberg Levels Based on Long Range Weakly Bound Molecular Ions[RL]}

Molecular ion potentials correlating with a ground state atom and a ground state ion do not necessarily have only a strongly bound potential. The "one electron" molecular ions like $\mathrm{Na}_{2}{ }^{+}$, have a strongly bound potential and a weakly bound potential. [74] presents the calculation of the bonding $\mathrm{X}^{2} \Sigma_{\mathrm{g}}{ }^{+}$potential with $\mathrm{D}_{\mathrm{e}}=$ $7905 \mathrm{~cm}^{-1}$ and $\mathrm{R}_{\mathrm{e}}=6.24 \mathrm{a}_{\mathrm{o}}$ and the antibonding ${ }^{2} \Sigma_{\mathrm{u}}{ }^{+}$potential with $\mathrm{D}_{\mathrm{e}}=43.9 \mathrm{~cm}^{-1}$ and $\mathrm{R}_{\mathrm{e}}=25 \mathrm{a}_{\mathrm{o}}$ (from attractive charge-induced dipole and dispersion interactions). While Rydberg series based on short range chemically bound molecular ions are well known and discussed in Section IV., to the best of my knowledge, there have been no serious attempts to explore such Rydberg series based on weakly bound long range states of molecular ions. Ultracold techniques, as in [75], allow vertical transitions, e.g. from long range levels, formed near dissociation to two ground state atoms by ultracold photoassociation in $\mathrm{Rb}_{2}$ or $\mathrm{KRb}$, to reach the traditional $u p$ and away Rydberg levels discussed in this section and in the next two sections on other types of Rydberg levels.

\section{Long Range Rydberg Levels Based on Atomic Ion Pairs[HR]}

It has long been known that atomic ion pairs were important for the description of the potential energy curves for many molecules [76], including even the lowest potential curves for the alkali metal hydrides [77]. See, for example, Figure 1 in [78] and for LiH Figure 171 in [27]. For atoms with a positive electron affinity, the atomic ion pair asymptote lies below the asymptote of a neutral atom plus an atomic ion and free electron. The experiments of [78] showed the strong influence of the $1 / \mathrm{R}$ ion pair potential on a variety of potentials of various excited ${ }^{1} \Sigma_{\mathrm{g}}{ }^{+}$states of $\mathrm{Na}_{2}$, in accord with the theory of $[79,80]$ and many later calculations (see also [82] and references therein). Recent work at UConn [80] has focused on $\mathrm{Rb}_{2}$,

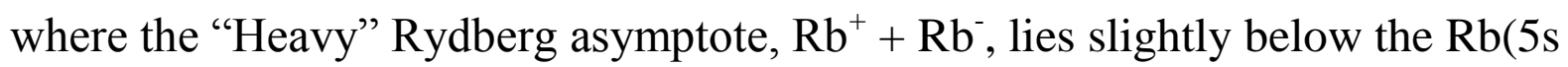
$\left.{ }^{2} \mathrm{~S}\right)+\mathrm{Rb}\left(8 \mathrm{p}{ }^{2} \mathrm{P}_{1 / 2}\right)$ asymptote. In particular, the $2^{1} \Sigma_{\mathrm{g}}{ }^{+}$and $3^{1} \Sigma_{\mathrm{u}}{ }^{+}$states have been and are being studied to better understand a portion of the ion pair potential [81, 82]. 
It is clear that there will be many interactions between the ion pair potentials and the other types of Rydberg potentials as a function of both $\mathrm{E}$ and R. For example, for the last class of long range Rydberg states discussed below in the next Section VII., there should be especially strong long range interactions for those states correlating to asymptotes with a single excited atom at low $\mathrm{n}$ (say $<8$ for $\mathrm{Rb}_{2}$ ) with the ion pair states we have just discussed in this section.

\section{VIII.Long Range Rydberg Levels Based on an Atom @ a Rydberg Atom[@R]}

Studies of a relatively new class of potential energy curves has arisen when a ground state atom is embedded inside (@) a much larger Rydberg atom. Greene et al.[83] showed that this "bond" arises from the attraction between the Rydberg electron and the embedded ground state atom. The Rydberg electron can have any allowed orbital angular momentum 1, but our focus in current experiments has been on s, p, and d electrons. For an s Rydberg electron, the potential can be reasonably approximated by the "Fermi pseudopotential" equation

$$
\mathrm{V}_{\mathrm{s}}(\mathrm{R})=2 \pi \mathrm{a}_{\mathrm{s}}(\mathrm{R})\left|\psi_{\mathrm{ns}}(\mathrm{R})\right|^{2}
$$

where $a_{s}$ is the triplet s-wave scattering length for the Rydberg $\mathrm{e}^{-}$from the ground state atom as a function of $\mathrm{R}$ and $\psi_{\mathrm{ns}}(\mathrm{R})$ is the wavefunction of the atomic ns state under consideration. Bound states in such potentials have been observed for high n, e.g. 35s [84], where the wells are extremely shallow and extremely far away at 500-2000 $\mathrm{a}_{0}$ !

We are studying the $p$ Rydberg electron states for low $n=7-12$, [75, 85], for which an additional $p$-wave term in the potential is needed. For the $7 p$ case, we have an ab initio potential with a minimum $\sim 160 \mathrm{~cm}^{-1}$ deep at an internuclear distance of $\sim 35 \mathrm{a}_{\mathrm{o}}$. Vibrational levels in this potential overlap well with the outermost lobe of the wavefunction for a long range vibrational level $(v "=35)$ in the a ${ }^{3} \Sigma_{\mathrm{u}}{ }^{+}$state produced selectively by ultracold photoassociation. Using the same long range $v "=35$ level, we then study the higher np states and observe spectra 
consistent with multiple extrema predicted by the "Fermi pseudopotential" potential [83]. We plan to use higher a state levels up to perhaps v" $=39$ (outer turning point $\sim 80 \mathrm{a}_{\mathrm{o}}$ ) to observe the predicted outermost wells for $10 \mathrm{p}, 11 \mathrm{p}$, and $12 \mathrm{p}$ at roughly 60,90 , and $120 \mathrm{a}_{\mathrm{o}}$.

Ultimately it may also be possible to study the interaction regions where two or more of the four types of Rydberg states listed here will mix with one another. It will be fascinating to behold.

\section{ACKNOWLEDGMENTS}

I especially appreciate the inspiration and understanding of potential energy curves I received from interactions with Dudley Herschbach, Alex Dalgarno, Dick Bernstein, Bob Le Roy, Paul Julienne, Moe Krauss, Fred Mies, Goran Pichler, Bob Field, Li Li, Ken Sando, Paul Kleiber, Marjatta Lyyra, John Bahns, He Wang, Warren Zemke, Olivier Dulieu, Eberhardt Tiemann, Rudolf Vidal, Ed Chang, Robin Côté, Winthrop Smith, Jin-Tae Kim, and especially Ed Eyler and Phil Gould, in semihistorical order. I have been fortunate to have worked with many other outstanding scientists on many other topics, some including those referenced in this article, and have been very fortunate to have had support from NSF(current), DOE, AFOSR, NASA, ONR, PRF, NATO, NRC, and other funding sources. My greatest support has always come from my wonderful wife, Mauricette.

\section{REFERENCES}

[1] H. E. Roscoe and A. Schuster, Prof. Roy. Soc. London 22, 362 (1874).

[2] W. C. Stwalley, J. Chem. Phys. 63, 3062 (1975).

[3] R. Krems, W. C. Stwalley, and B. Friedrich, editors, Cold Molecules: Theory, Experiment, Applications, Taylor and Francis, New York, 2009. 
[4] W. C. Stwalley, P. L. Gould, and E. E. Eyler, "Ultracold Molecule Formation by Photoassociation", in R. Krems, W. C. Stwalley, and B. Friedrich, editors, Cold Molecules: Theory, Experiment, Applications, Taylor and Francis, New York, 2009, p. 169.

[5] W. C. Stwalley and H. Wang, J. Mol. Spectrosc. 195, 194 (1999).

[6] J. T. Bahns, P. L. Gould, and W. C. Stwalley, Adv. At. Mol. Opt. 42, 171 (2000).

[7] J. T. Kim, B. Kim, and W. C. Stwalley, Analysis of the Alkali Metal Diatomic Spectra: Using Molecular Beams and Ultracold Molecules, ebook- Institute of Physics Series in Concise Physics, 2014.

[8] J. L. Dunham, Phys. Rev. 41, 721 (1932).

[9] G. Simons, R. G. Parr, and J. M. Finlan, J. Chem. Phys. 59, 3229 (1973).

[10] A. J. Thakkar, J. Chem. Phys. 62, 1693 (1975).

[11] J. E. Kilpatrick, J. Chem. Phys. 30, 801 (1959).

[12] O. Oldenburg, Z. Phys. 66, 563 (1929).

[13] R. Rydberg, Z. Phys. 73, 376 (1931).

[14] O. Klein, Z. Phys. 76, 226 (1932).

[15] A. L. G. Rees, Proc. Phys. Soc. (London) 59, 998 (1947).

[16] W. M. Kosman and J. Hinze, J. Mol. Spectrosc. 56, 93 (1975).

[17] C. R. Vidal and H. Scheingraber, J. Mol. Spectrosc. 65, 46 (1977).

[18] C. R. Vidal and W. C. Stwalley, J. Chem. Phys. 77, 883 (1982).

[19] R. J. Le Roy, Can. J. Phys. 52, 246 (1974). 
[20] J. Y. Seto, R. J. Le Roy, J. Vergés, and C. Amiot, J. Chem. Phys. 113, 3067 (2000).

[21] R. J. Le Roy, Y. Huang, and C. Jary, J. Chem. Phys. 125, 164310 (2006).

[22] R. J. Le Roy, N. S. Dattani, J. A. Coxon, A. J. Ross, P. Crozet, and C. Linton, J. Chem. Phys. 131, 204309 (2009).

[23] R. J. Le Roy, in Molecular Spectroscopy I, R. F. Barrow, D. A. Long, and D. J. Millin, editors, Chemical Society, London, 1973, p. 113.

[24] See http://leroy.uwaterloo.ca/programs.html.

[25] R. B. Bernstein, Phys. Rev. Lett. 16, 385 (1966).

[26] R. S. Mulliken, J. Phys. Chem. 41, 5 (1937).

[27] G. Herzberg, Molecular Spectra and Molecular Structure: I. Spectra of Diatomic Molecules, D. Van Nostrand, Princeton (1950).

[28] P. J. Hay, W. R. Wadt, L. R. Kahn, and F. W. Bobrowicz, J. Chem. Phys. 69, 984 (1978).

[29] S. Sasmal, H. Pathak, M. K. Nayak, N. Vaval, and S. Pal, J. Chem. Phys. 144, 124307 (2016).

[30] W. C. Stwalley, A. Niehaus, and D. R. Herschbach, "Velocity Dependence of the Total Cross Section for Scattering of Hydrogen Atoms from Mercury", Proceedings of the Vth International Conference on the Physics of Electronic and Atomic Collisions, Nauka Press, Leningrad, 1967, p. 639.

[31] W. C. Stwalley and H. L. Kramer, J. Chem. Phys. 49, 5555 (1968).

[32] B. Ji, C.-C. Tsai, and W. C. Stwalley, Chem. Phys. Lett. 236, 242 (1995).

[33] W. C. Stwalley, A. Niehaus, and D. R. Herschbach, J. Chem. Phys. 63, 3081 (1975). 
[34] K. A. Köhler, R. Feltgen, and H. Pauly, Phys. Rev. A 15, 1407 (1977).

[35] Y. H. Uang and W. C. Stwalley, J. Chem. Phys. 76, 5069 (1982).

[36] W. C. Stwalley, Chem. Phys. Lett. 6, 600 (1970).

[37] W. C. Stwalley, J. Chem. Phys. 54, 4517 (1971).

[38] K. C. Li and W. C. Stwalley, J. Chem. Phys. 59, 4423 (1973).

[39] W. C. Stwalley, J. Chem. Phys. 65, 2038 (1976).

[40] W. C. Stwalley, Chem. Phys. Lett. 6, 241 (1970).

[41] W. C. Stwalley, J. Chem. Phys. 58, 536 (1973).

[42] D. Wang, J. Qi, M. F. Stone, O. Nikolayeva, B. Hattaway, S. D. Gensemer, H. Wang, W. T. Zemke, P. L. Gould, E. E. Eyler, and W. C. Stwalley, Eur. Phys. J. D 31, 243005 (2004).

[43] R. J. Le Roy and R. B. Bernstein, J. Chem. Phys. 52, 3869 (1970).

[44] W. C. Stwalley, Contemp. Phys. 19, 65 (1978).

[45] W. C. Stwalley, Chem. Phys. Lett. 19, 337 (1973).

[46] W. C. Stwalley, J. Chem. Phys. 58, 3867 (1973).

[47] A. R. Janzen and R. A. Aziz, J. Chem. Phys. 103, 9626 (1995).

[48] G. Herzberg, J. Mol. Spectrosc. 33, 147 (1970).

[49] W. T. Zemke and W. C. Stwalley, J. Phys. Chem. 97, 2053 (1993).

[50] W. T. Zemke and W. C. Stwalley, J. Chem. Phys. 100, 2661 (1994). 
[51] W. T. Zemke, C.-C. Tsai, and W. C. Stwalley, J. Chem. Phys. 101, 10382 (1994).

[52] G. Zhao, W. T. Zemke, J. T. Kim, B. Ji, H. Wang, J. T. Bahns, W. C.

Stwalley, L. Li, A. M. Lyyra, and C. Amiot, J. Chem. Phys. 105, 7976 (1996).

[53] W. T. Zemke and W. C. Stwalley, J. Chem. Phys. 111, 4956 (1999).

[54] W. T. Zemke and W. C. Stwalley, J. Chem. Phys. 111, 4962 (1999).

[55] W. C. Stwalley, Y. H. Uang, and G. Pichler, Phys. Rev. Lett. 41, 1164 (1978).

[56] K. Niemax and G. Pichler, J. Phys. B 8, 179 (1975).

[57] H. Wang, P. L. Gould, and W. C. Stwalley, Z. Phys. D 36, 317 (1996).

[58] L. P. Ratcliff, M. E. Wagshul, P. D. Lett, S. L. Rolston, and W. D. Phillips, J. Chem. Phys. 101, 2638 (1994).

[59] H. Wang, J. Li, X. T. Wang, C. J. Williams, P. L. Gould, and W. C. Stwalley, Phys. Rev. A 55, R1569 (1997).

[60] X. T. Wang, H. Wang, P. L. Gould, W. C. Stwalley, E. Tiesinga, and P. S. Julienne, Phys. Rev. A 57, 4600 (1998).

[61] R. A. Cline, J. D. Miller, and D. J. Heinzen, Phys. Rev. Lett. 73, 632 (1994).

[62] W. C. Stwalley, M. Bellos, R. Carollo, J. Banerjee, and M. Bermudez, Mol. Phys. 110, 1739 (2012).

[63] W. C. Stwalley, J. Chem. Phys. 56, 2485 (1972).

[64] K. K. Verma, J. T. Bahns, A. R. Rajaei-Rizi, W. C. Stwalley, and W. T. Zemke, J. Chem. Phys. 78, 3599 (1983). 
[65] M. E. Koch, W. C. Stwalley, and C. B. Collins, Phys. Rev. Lett. 42, 1052, (1979).

[66] M. A. Bellos, D. Rahmlow, R. Carollo, J. Banerjee, O. Dulieu, A. Gerdes, E. E. Eyler, P. L. Gould, and W. C. Stwalley, Phys. Chem. Chem. Phys. 13, 18880 (2011).

[67] J. T. Kim, D. Wang, E. E. Eyler, P. L. Gould, W. C. Stwalley, New J. Phys. 11, 055020 (2009).

[68] M. Masters, J. Huennekens, W. T. Liu, L. Li, A. M. Lyyra, K. Sando, V. Zafiropulos, and W. C. Stwalley, J. Chem. Phys. 92, 5801 (1990).

[69] K. P. Huber and G. Herzberg, Molecular Spectra and Molecular Structure IV. Constants of Diatomic Molecules, Van Nostrand Reinhold, New York (1979).

[70] R. S. Mulliken, Accounts of Chemical Research 9, 7 (1976).

[71] H. Wang, W. C. Stwalley, and A. M. Lyyra, J. Chem. Phys. 96, 7965 (1992).

[72] C.-C. Tsai, J. T. Bahns, and W. C. Stwalley, Chem. Phys. Lett. 236, 553 (1995).

[73] E. S. Chang, J. Li, J. Zhang, C.-C. Tsai, J. T. Bahns, and W. C. Stwalley, J. Chem. Phys. 111, 6247 (1999).

[74] C. Cerjan, K. K. Docken, and A. Dalgarno, Chem. Phys. Lett. 38, 401 (1976).

[75] M. A. Bellos, R. Carollo, J. Banerjee, E. E. Eyler, P. L. Gould, and W. C. Stwalley, Phys. Rev. Lett. 111, 053001 (2013).

[76] R. S. Mulliken, Phys. Rev. 50, 1017 (1936).

[77] R. S. Mulliken, Phys. Rev. 50, 1028 (1936). 
[78] C.-C. Tsai, J. T. Bahns, H. Wang, W. C. Stwalley, and A. M. Lyyra, Phys. Rev. Lett. 71, 1152 (1993).

[79] A. Sanli, B. Beser, J. R. Edwardson, S. Magnier, E. H. Ahmed, and A. M. Lyyra, J. Chem. Phys. 143, 104304 (2015).

[80] A. Kirrander, S. Rittenhouse, M. Ascoli, E. E. Eyler, P. L. Gould, and H. Sadeghpour, Phys. Rev. A 87, 031402 (2013)

[81] M. Ascoli, Ph.D. thesis, Dept. of Physics, University of Connecticut (2015).

[82] J. Yang, Y. Guan, W. Zhao, Z. Zhou, X. Han, J. Ma, V. B. Sovkov, V. S. Ivanov, E. H. Ahmed, A. M. Lyyra, and X. Dai, J. Chem. Phys. 144, 024308 (2016).

[83] C. H. Greene, E. L. Hamilton, H. Crowell, C. Vadla, and K. Niemax, Phys. Rev. Lett. 97, 233002 (2006) and references therein.

[84] V. Bendowsky, B. Butscher, J. Nipper, J. P. Shaffer, R. Löw, and T. Pfau, Nature (London) 458, 1005 (2009).

[85] M. A. Bellos, R. Carollo, J. Banerjee, M. Ascoli, A.-R. Allouche, E. E. Eyler, P. L. Gould, and W. C. Stwalley, Phys. Rev. A 87, 012508 (2013). 
Graphical Abstract of Topics Defined in Text

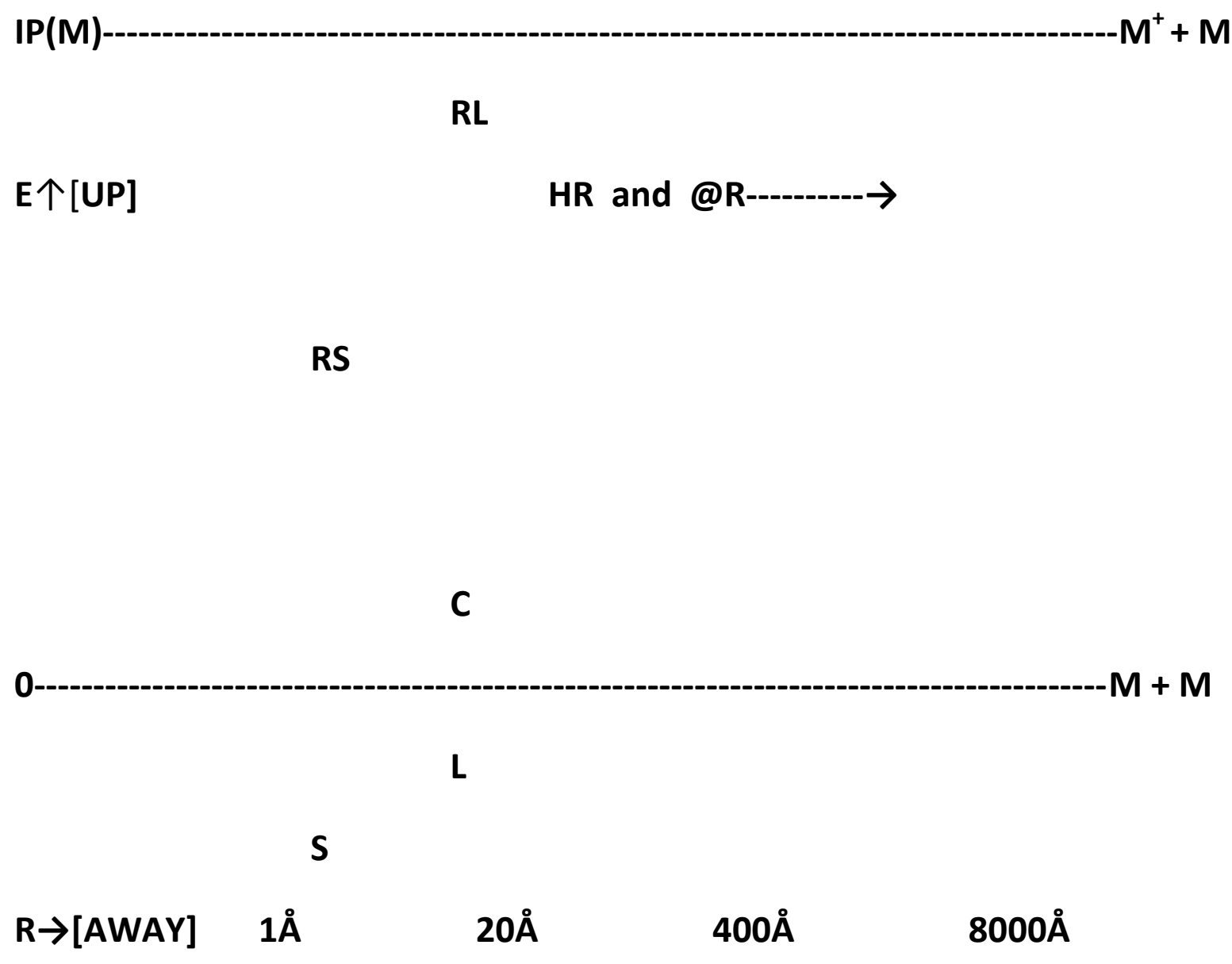

